\title{
Perfil del turista ecológico, aspectos sociodemográficos, expectativas y actividades del ecoturista en España
}

\section{Profile of the ecological tourist, sociodemographic aspects, expectations and activities of the ecotourist in Spain}

José Alberto Crespo Jareño?

Resumen: Esta investigación pretende conocer el perfil del turista residente en España que tiene la intención de realizar ecoturismo o turismo ecológico, así como conocer los principales servicios que éstos demandan y las actividades de interés. España es un país que cuenta con una extensa protección de su territorio y a la vez es uno de los países donde el turismo, y en concreto el turismo de naturaleza tiene cifras muy importantes. Se empleó una metodología cuantitativa a través de una encuesta realizada en línea, se realizaron 403 entrevistas a través de una base de datos electrónica de una empresa especializada trabajando sobre aspectos demográficos, socioeconómicos y sobre los servicios ecoturísticos. Entre otros resultados se aprecia que el perfil del turista de naturaleza es hombre, mayor de 45 años, pose estudios universitarios, vive en localidades de más de diez mil habitantes y tiene un salario entorno a la media. El estudio supone una oportunidad para los operadores turísticos, porque facilita el diseño de planes y estrategias de comunicación y marketing para de cara a los futuros consumidores.

Palabras clave: perfil del turista, España, ecoturismo, residentes.
Abstract: This research aims to know the profile of the tourist resident in Spain who intends to carry out ecotourism or ecological tourism, as well as to know the main services that these demand and the activities of interest. Spain is a country that has extensive protection of its territory and at the same time is one of the countries where tourism, and specifically nature tourism has very important figures. We used a quantitative methodology through an online survey conducted 403 interviews through an electronic database of a specialized company working on demographic, socioeconomic and ecotourism services. Among other results it can be seen that the profile of the nature tourist is male, over 45 years old, has university studies, lives in towns of more than ten thousand inhabitants, and has a salary around the average. The study is an opportunity for tour operators because it facilitates the design of communication and marketing plans and strategies for future consumers.

Key Words: tourist profile, Spain, ecotourism, residents.

(Presentado: 30 de octubre de 2019. Aceptado: 20 de noviembre de 2019) ${ }^{1}$ Doctor en Economía y Empresa, Universidad de Castilla-La Mancha, España. Miembro del Colegio de políticas y sociología de Castilla-La Mancha. Gestor del Estado en
el Instituto de la Juventud de España. crespojaQqoutlook.es 


\section{INTRODUCCIÓN}

La actividad turística es una de las principales industrias económicas a nivel mundial, si bien, se han ido produciendo cambios significativos debido a que se ha pasado de un turismo de masas a un turismo alternativo que respeta el medioambiente y donde el turista adquiere nuevas experiencias en contacto con la naturaleza, la cultura y los residentes de un territorio. En los últimos años han aparecido nuevas formas de turismo más sostenibles, donde existen elementos relacionados con el descanso, el disfrute y la protección del medioambiente o el conocimiento de la cultura local, a través de políticas que favorecen el desarrollo sostenible, debido a que la sociedad está cada día más concienciada sobre los impactos negativos hacia la naturaleza, tanto por motivos económicos como culturales y sociales.

La oportunidad de conocer el perfil de las personas que hacen turismo ecológico o ecoturismo en España, radica en la escasez de estudios globales que busquen el perfil de los turistas ecológicos en el ámbito iberoamericano.

En este exhaustivo repaso, Kim (2017) señala que no hay muchos estudios que fijen como sujeto de estudio al ecoturista, porque, por un lado, el concepto sobre qué es el ecoturismo no está claro, por tanto, es difícil investigar a los sujetos que lo realizan y, por otro lado, porque la industria no ha mostrado interés, dado el bajo número de personas que hacen ecoturismo. Esto indica la necesidad y la oportunidad de seguir aproximándonos en el conocimiento de estos perfiles.

Según Europarc (2016), España cuenta con un 13\% de su territorio como espacios naturales protegidos, cifra que se amplía por si consideramos los territorios que incluye la Red Natura 2000, siendo en ese caso del $27 \%$ del territorio nacional. Tan solo los 15 parques con la categoría de nacionales generan más de diez millones de visitas al año (Cobo y Aparicio, 2014).

\section{MARCO TEÓRICO}

\section{El ecoturismo}

La preocupación por el medioambiente también ha cambiado el desarrollo del turismo como industria, y ha provocado la implosión del denominado turismo sostenible y del ecoturismo, que intentan conjugar los valores ambientales y el desarrollo del turismo (Dias, 2007; Puhakka y Siikamäki, 2012), y que actualmente crece tres veces más rápido que el turismo convencional, esperando ocupar un lugar más destacado en el mercado global de turismo (Das y Chatterjee, 2015). Así, la Organización Mundial del Turismo ha señalado que el $10 \%$ del turismo mundial se realiza a partir de motivaciones ecoturísticas (Pérez de la Heras, 1999) y que es de esperar un mayor incremento de este tipo de turismo frente al turismo de masas.

Weaver y Lawton (2007), señalan que el ecoturismo empezó a aparecer en los textos académicos internacionales en los años ochenta, y su importancia ha crecido exponencialmente en los últimos veinte años. El ecoturismo conjuga, según estos autores, tres criterios esenciales: el primero se trata de un turismo cuya primera atracción es la naturaleza, el segundo en él los visitantes se relacionan con el medio natural a través de la educación y, en tercer lugar, la experiencia turística debe enfocarse a través de prácticas que ecológica, cultural y económicamente sean sostenibles.

El termino ecoturismo, señala Jiménez (2009), toma luz de manos de la Conferencia de Naciones Unidas para el Medio Ambiente Humano de 1972, donde aparece el eco-desarrollo. El mexicano Héctor Ceballos Lascuráin (1987), definió inicialmente el ecoturimo:

"Viajar a visitar áreas naturales relativamente sin alteración, incluyendo las áreas protegidas con el fin de disfrutar, apreciar y estudiar los atractivos naturales (paisaje, flora y fauna silvestre), así como cualquier manifestación cultural del presente y del pasado, mediante un proceso que promueve la conservación, tiene bajo impacto negativo ambiental y cultural, propicia un involucramiento activo y socioeconómicamente benéfico de las comunidades locales" (p. 120).

\section{El ecoturista}

En el ámbito turístico, Dolnicar (2008) señala que cada turista es diferente, por lo que los destinos que prefiere son diferentes, sin embargo, estos pueden tener características comunes con otros. Este autor señala que la segmentación del mercado es una estrategia que implica que personas 
con similares características sean agrupadas. En la misma línea, Neuts, Romão, Nijkamp y Shikida (2016) señalan que el mercado turístico es heterogéneo, por lo que es necesario segmentar el mercado para concentrar en los grupos de interés las acciones que se idean desde el marketing por las organizaciones que realizan ecoturismo.

Para Beaumont (2011), para segmentar se necesita que los segmentos sean medibles, accesibles y sustanciales. Así una segmentación será eficaz si existe una facilidad para acceder a grupos distintos y relevantes (Molina, Martín-Consuegra, Esteban, Díaz, 2007). Santesmases (1999), realizó una clasificación de los criterios de la segmentación dividiendo en una tabla de cuatro entradas, entre criterios objetivos, subjetivos y entre criterios generales y subjetivos.

Tabla N N 1. Criterios de segmentación de los mercados.

\begin{tabular}{lll}
\hline & Generales & Específicos \\
\hline Objetivos & Demográficos: sexo, edad. & Estructura de consumo (grande, mediano, etc.) \\
& Socioeconómicos: renta, ocupación, & Uso del producto. \\
& Geográficos: región, hábitat, etc. & nivel de estudios, etc. \\
& & Fidelidad/lealtad a la marca /empresa. \\
& Tipo de compra: primera o repetición. \\
& Situaciones de compra. \\
Subjetivos & Lugar de compra. \\
& Personalidad: liderazgo, autoridad, autonomía. & Ventajas/beneficio buscado. \\
& Estilos de vida: centros de interés, opiniones, etc. & Actitudes. \\
& & Percepciones. \\
& Preferencias.
\end{tabular}

Fuente: Santesmases (1999).

La idea clásica de ecoturismo, como viaje especializado ligado a la naturaleza, pone el acento en las consideraciones sociodemográficas a la hora de segmentar el mercado. Siguiendo a los autores Meric y Hunt (1998), en su investigación sobre el ecoturista en Estados Unidos (Carolina del Norte) el ecoturista, una vez que se comporta como tal, lo que significa disfrutar de su viaje con un impacto ambiental mínimo, es un consumidor con ingresos más altos que la población en general, de mediana edad y con una educación superior a la media. No obstante, esta clasificación sociodemográfica, varía en los diferentes estudios, lo que ha provocado que parte de la academia considere que estos datos no son los más adecuados para realizar la diferenciación entre los ecoturistas y entre los que no lo son (Dolnicar y Long, 2009).

Puertas (2006), señala que el ecoturista tiene una característica propia o diferente al resto de turistas, dado que busca experiencias y actividades turísticas distintas a las habituales, donde la naturaleza es esencial y a su vez que no perjudiquen el medioambiente. En cuanto a los tipos de ecoturistas, siguiendo a Lindberg (1991), citado en Puertas (2006), estos visitantes pueden clasificarse en: ecoturistas ocasionales, que son aquellos que, dentro de un viaje, tienen previsto visitar algún espacio natural; los ecoturistas de hitos naturales, que son aquellos que huyen especialmente de los viajes habituales y quieren disfrutar de los espacios naturales; los ecoturistas interesados, que buscan aspectos de interés en la naturaleza, y los ecoturistas científicos, cuyo interés es más profesional que recreativo. Pérez, Salinas y Pérez (2012), consideran que el ecoturista puede estudiar y disfrutar el paisaje, desde una perspectiva conservacionista.

Para Juric, Cornwell y Mather (2002), los ecoturistas son aquellos individuos interesados no solo en la conservación cultural o ecológica, sino en el aprendizaje y la vivencia de experiencias nuevas y diferentes en el medio natural. Nel.lo y LLanes (2016), asegura que los visitantes cuyo rasgo principal es su actitud hacia el medio natural son en la actualidad un $10 \%$ de las personas que viajan, aunque existen pocos estudios que analicen los perfiles de este tipo de turistas. 
El perfil más habitual, siguiendo a Nel.lo y LLanes (2016), es el de una persona con estudios, activo, educado, inteligente, curioso, aventurero y con conocimientos previos del lugar que visita. El ecoturista busca lo nuevo, desea un intercambio cultural, aprendizaje y prefiere un servicio personalizado y de calidad. El ecoturista busca también la libertad, la exclusividad y la privacidad. En relación con las actividades que desarrolla durante su viaje, destacan la observación de aves, la fotografía, los estudios botánicos, el submarinismo de observación, frente a las actividades generalistas o recreativas del turista tradicional. En cuanto a su manera de comportarse, llama la atención que eligen desplazarse en pequeños grupos con guías especializados. Su forma de compra suele ser mayoritariamente a través de paquetes turísticos con un elevado gasto por día.

En relación a las actividades que realizan los ecoturistas, Nel.lo y Llanes (2016), citan un estudio de WWF en 1998 realizado en América Latina y el Caribe, donde señalan que las principales actividades en el medio natural que hicieron los visitantes, como forma de clasificación, fueron: la observación de aves (58\%), observación de fauna silvestre (55\%), paseos en barca (42\%), botánica (31\%), senderismo (28\%), conocimiento de culturas locales (25\%), caminatas por la selva (23\%) y montañismo (22\%).

\section{METODOLOGÍA}

Se planteó una investigación en línea en España a través de un cuestionario en colaboración de la base de datos de una compañía de investigación de mercados. Las características iniciales de la muestra son: a) Turistas que manifiestan su condición de ecoturista, b) Mayores de 15 años y c) Nacionales españoles residentes en España.

Con el fin de generar un filtro adecuado de la muestra, la primera premisa fue conseguir individuos ecoturistas, tras una explicación completa del concepto, en los siguientes términos "entendemos por ecoturista, aquella persona que ha realizado alguna de las siguientes actividades: haber visitado parques naturales, reservas, avistamiento de pájaros o ballenas, visitas a comunidades indígenas; haber realizado montañismo, trekking, senderismo, turismo étnico o indígena, safari, agroturismo, turismo rural, haber estado alojado en un hotel ecológico, etc., con respeto a la naturaleza, en favor de aprendizaje y que, al menos, hayan desarrollado alguna de una de estas actividades durante el último año, asimilando estos criterios a los empleados por las investigaciones en línea en materia de turismo específico”, basándonos en Han, Hwang y Lee (2016).

En primer lugar, hicimos el acopio y análisis de las referencias bibliográficas sobre ecoturismo, comportamiento del consumidor, con el objetivo de identificar la literatura más importante sobre estas materias, los métodos de investigación y los principales resultados de otras investigaciones, así como los espacios para desarrollar nuestro trabajo.

El cuestionario consta de las siguientes partes:

- Variables demográficas en las que hemos incluido: edad, sexo (hombre/mujer) y lugar de residencia (más/menos 10.000 habitantes).

- Variables socioeconómicas, donde se realizan preguntas sobre los ingresos mensuales salariales y del hogar familiar, así como del nivel educativo.

Posteriormente aplicamos un pretest para validar el cuestionario, a través de expertos del sector y consumidores ecoturistas. Se distribuyó el cuestionario original entre 31 visitantes españoles, del Parque Natural de las Lagunas de Ruidera, el día 15 de abril de 2017. La finalidad era comprobar el buen entendimiento del cuestionario y conocer el tiempo empleado en su cumplimentación. A lo largo de esa jornada, los cuestionarios fueron cumplimentados por 16 mujeres y 15 varones, con edades superiores a 15 años. Su cumplimentación no aportó dudas al conjunto de las personas que realizaron el cuestionario, salvo lo que tuvo que ver con la extensión del cuestionario y la duración.

Las principales dificultades del cuestionario según los estudiantes fueron:

- En la pregunta 7 se retiró el término Snorkeling por su desconocimiento y se optó por el genérico buceo.

- Con relación a las variables económicas surgieron algunas dudas con el concepto bruto o neto de los ingresos.

En esta investigación optamos por una encuesta en línea a un panel de consumidores de la empresa de investigación de mercados Netquest, que disponible de panelistas 
en España. Con ello intentamos acercarnos más al grupo de consumidores en el que estamos interesados También en el formato en línea se busca un mayor interés y participación de los encuestados. Por otro lado, consideramos que la encuesta en línea el anonimato de los entrevistados es más amplio y pensamos que puede facilitar la autenticidad de las respuestas, así como mejora la confortabilidad del usuario para rellenarla.

\section{Tabla Nº 2. Ficha técnica de la investigación.}

\section{Ficha técnica}

Fecha de realización

Método de recogida de datos

Técnicas de análisis de datos

Universo

Muestra

Error muestral

Nivel de la confianza

Procedimiento de muestreo

Metodología

Escalas

Medidas de control

Lugar de realización oct-17

Formato en línea con apoyo de empresa.

Técnicas univariantes: descriptivos y frecuencia.

Población turista española.

403 turistas nacionales de España.

$95 \%, 5 \%$ por muestra.

$\mathrm{p}=\mathrm{q} 0,50$

Por cuotas: género y edad de turistas.

Cuantitativa.

Likert de 5 puntos.

Pretest: 15 abril 2017 Ruidera, Ciudad Real (España).

España.
Nuestra muestra está basada en los resultados de la encuesta de movimientos turísticos de los españoles (Familitur), que realizo en 2016 el Instituto Nacional de Estadística (2016), que recoge datos relativos a los viajes que realizan los residentes en España. Hemos realizado el cál- culo por género y edad. El cuestionario comienza con las preguntas de edad y sexo, y con una pregunta más amplia que pretende ejercer un filtro en relación con las actividades ecoturísticas en un periodo de tiempo previo. Véase la Tabla 3.

\section{Tabla Nº 3. Pregunta filtro para cuestionario.}

\begin{tabular}{ll}
\hline Ítem & Medida \\
\hline ¿Cuáles de estas actividades has realizado en & Visitar parques naturales, reservas, safaris, \\
el último año? & avistamiento de pájaros o ballenas. \\
& Montañismo, trekking, senderismo. \\
& Turismo étnico o indígena. \\
& Agroturismo o turismo rural. \\
& Visitar una gran ciudad. \\
& Ir a un concierto o festival de música. \\
& Turismo de sol y playa. \\
& Visitar lugares culturales o patrimoniales. \\
& Ninguna de las anteriores.
\end{tabular}

\section{RESULTADOS}

Esta investigación se enfocó en distintos apartados, por un lado, para conocer los aspectos demográficos y socioeconomicos. Podemos observar que ambos géneros (hombres y mujeres) están representados en la muestra por partes iguales, conforme se aprecia en la Tabla 4.
Tabla № 4. Distribución de la muestra por género.

\begin{tabular}{ll} 
& España \\
Hombre & 208 \\
Mujer & 195 \\
Total & 403 \\
\hline
\end{tabular}


En la muestra fueron 208 varones y 195 mujeres las que completaron el cuestionario. El p-valor para la prueba del chi-cuadrado de Pearson nos revela que la distribución entre géneros es equitativa.

\section{Tabla № 5. Distribución de la muestra por país y edad.}

\begin{tabular}{lcc}
\hline Tramo de edad & $\begin{array}{c}\text { España } \\
\text { Frecuencia }\end{array}$ & $\begin{array}{c}\text { España } \\
\text { Porcentaje }\end{array}$ \\
$16-29$ & 82 & $20,35 \%$ \\
$30-44$ & 134 & $33,3 \%$ \\
$45-64$ & 141 & $35 \%$ \\
$>=65$ & 46 & $11,4 \%$ \\
Total & 403 & $100 \%$ \\
\hline
\end{tabular}

Encontramos que, entre los encuestados, en España el $81 \%$ vive en ciudades de más de 10.000 habitantes, En relación con los turistas que viven en municipios de menos de 10.000 habitantes, en España el porcentaje es del 19\%.

\section{Tabla Nº 6. Distribución de la muestra agru-} pada por residencia.

\begin{tabular}{|c|c|c|}
\hline & $\begin{array}{c}\text { España } \\
\text { Frecuencia }\end{array}$ & $\begin{array}{c}\text { España } \\
\text { Porcentaje }\end{array}$ \\
\hline $\begin{array}{l}\text { Localidad con más } \\
\text { de } 10.000 \text { habitantes }\end{array}$ & 326 & $81 \%$ \\
\hline $\begin{array}{l}\text { Localidad con menos } \\
\text { de } 10.000 \text { habitantes }\end{array}$ & 77 & $19 \%$ \\
\hline Total & 703 & $100 \%$ \\
\hline
\end{tabular}

En relación con la situación laboral por país (véase la Tabla 7), encontramos que el porcentaje de empleados es de un $58,56 \%$. Por el contrario, el número de trabajadores autónomos o independientes es ,96\% de ecoturistas españoles. Por otro lado, los desempleados, suponen un 5,96\%. En relación con los empresarios, la cifra es $0,99 \%$.

En cuanto a las personas que se dedican a las labores del hogar, las cifras son similares, $2,33 \%$ para $2,71 \%$. Los estudiantes son más numerosos en la muestra $8,44 \%$. Por último, en relación con los jubilados, éstos son un $13,9 \%$ en la muestra.
Tabla N 7. Distribución de la muestra agrupada por situación laboral y país. P-valor

\begin{tabular}{lcc}
\hline & $\begin{array}{c}\text { España } \\
\text { Frecuencia }\end{array}$ & $\begin{array}{c}\text { España } \\
\text { Porcentaje }\end{array}$ \\
Empleado/a & 236 & $58,56 \%$ \\
Independiente / Autónomo & 24 & $5,96 \%$ \\
Desempleado/a & 34 & $8,44 \%$ \\
Empresario/a & 4 & $0,99 \%$ \\
Labores del hogar & 9 & $2,23 \%$ \\
Estudiante & 34 & $8,44 \%$ \\
Jubilado/a & 56 & $13,90 \%$ \\
Otros & 6 & $1,49 \%$ \\
Total & 403 & $100,00 \%$ \\
\hline
\end{tabular}

En relación a los estudios, véase tabla 8, podemos señalar que el colectivo más numeroso es el de los titulados universitarios, siendo en España un 45,66\%. En relación con las personas con titulaciones de postgrado o superior, en España el porcentaje es de un 15,38\% de la muestra. En relación con las personas con titulaciones de Técnico Superior son del $15,88 \%$. El porcentaje de personas que no tienen estudios o éstos son de nivel básico es del $2,48 \%$. Por último, las personas con titulación media son, en el caso de España, el 20,60\% de la muestra.

\section{Tabla N 8. Distribución de la muestra por país y estudios.}

\begin{tabular}{lcc}
\hline & España & España \\
Frecuencia & Porcentaje \\
Sin estudios & 0 & $0,00 \%$ \\
Nivel básico & 10 & $2,48 \%$ \\
Nivel medio & 83 & $20,60 \%$ \\
Técnico Superior & 64 & $15,88 \%$ \\
Nivel Universitario & 184 & $45,66 \%$ \\
Postgrado/D & 62 & $15,38 \%$ \\
Total & 403 & $100,00 \%$ \\
\hline
\end{tabular}

Con relación a la autoubicación por parte de los ecoturistas encuestados y su nivel de ingresos en España -con un salario mensual medio de 1.500 euros-, encontramos que hay una parte importante de la muestra, el $11,47 \%$, que decide no facilitar esos datos, según se muestra en la Tabla 9. Entre los que sí los facilitan, el grupo mayoritario 
son las personas que se autoubican cercanas a esa cifra (31,025\%), por encima de ella, el $24,57 \%$ y muy por encima de ella, el 1,74\%. En el lado opuesto encontramos a las personas que no cuentan con ingresos propios, que representan el $8,44 \%$ y a los que se autoubican muy lejanos a la cifra media, $22,83 \%$.

Tabla Nº 9. Distribución de la muestra de España por salario mensual.

\begin{tabular}{lcc}
\hline & Frecuencia & Porcentaje \\
Sin ingresos propios & 34 & $8,44 \%$ \\
Muy por debajo de la cifra & 92 & $22,83 \%$ \\
Cercano/a a la cifra & 125 & $31,02 \%$ \\
Por encima de la cifra & 99 & $24,57 \%$ \\
Muy por encima de la cifra & 7 & $1,74 \%$ \\
Prefiero no contestar & 46 & $11,41 \%$ \\
Total & 403 & $100 \%$ \\
\hline
\end{tabular}

Con relación al ingreso bruto total de los hogares en España, según se aprecia en la Tabla 10, encontramos un elevado porcentaje de no respuestas (15,1\%). Entre los que sí responden, el grupo más numeroso son los hogares que tienen ingresos brutos totales superiores a 2.700 euros mensuales, que suponen $9,5 \%$, muy cerca se encuentran aquellos que ingresan entre 2.001 y 2.700 euros, que representan un $9,4 \%$ y los que dicen tener ingresos entre 1.301 y 2.000 euros, que son un $8,3 \%$ de las personas encuestadas. En cifras más bajas encontramos a las personas cuyos ingresos brutos por hogar están entre 901 y 1.300 euros $(5,1 \%)$ y los que señalan que tienen ingresos por hogar entre 0 y 900 euros, que suponen un colectivo del $2,5 \%$ de los encuestados.

Tabla N 10. Distribución de ingresos brutos por hogar: España.

De 0 a 900 euros mensuales Frecuencia Porcentaje De 901 a 1.300 euros mensuales 20 $4,96 \%$ 1.301 a 2.000 euros mensuales 41 67

2.001 a 27.00 euros mensuales 76 Más de 2.700 euros mensuales 77 Prefiero no contestar

Total
$16,63 \%$

$18,86 \%$

$19,11 \%$

$30,27 \%$

$100 \%$
En cuanto a la pregunta, ya empleada por Juric et al. (2002), con quién se hace el turismo ecológico (véase la Tabla 11) los encuestados muestran que viajan de forma independiente en un $69,98 \%$, a través de un tour organizado en un 0,99\% y conjugando ambas formas, un $29,03 \%$.

Tabla № 11. Estadísticos sobre la forma de realizar los viajes por país.

\begin{tabular}{lcc}
\hline & España & España \\
frecuencia & porcentaje \\
De forma independiente & 282 & $69,98 \%$ \\
A través de un tour organizado & 4 & $0,99 \%$ \\
Ambas, & 117 & $29,03 \%$ \\
Total & 403 & $100 \%$ \\
\hline
\end{tabular}

El alojamiento durante el viaje es una de las cuestiones esenciales a la hora de realizar turismo. En nuestro caso se dio la opción de poder señalar varios tipos de alojamiento más habitual. En el caso los alojamientos rurales son los más empleados (véase tabla 12).

\section{Tabla No 12. Estadísticos sobre el alojamien- to durante el viaje por país.}

Hotel España frecuencia

hostal/albergue/hostel 151

hotel ecológico

191

camping/carpa/cabañas 43

alojamiento rural

146

267

casa de amigos o conocidos

99

otros

18

En este caso, la primera pregunta que se formula quiere recoger información sobre la manera de viajar, solo o acompañado. Se trata de una pregunta de opción múltiple, recogida en la Tabla 13, ya empleada por Juric et al. (2002). Señala que, la forma más frecuente es el viaje en pareja.

Tabla No 13. Estadísticos sobre las personas con las que se viaja por países.

\begin{tabular}{lc}
\hline & España Frecuencia \\
Solo & 51 \\
Pareja & 245 \\
Familia & 144 \\
Amigos & 193 \\
otros & 6 \\
\hline
\end{tabular}


La pregunta que se formula quiere recoger datos relativos a las actividades que pueden realizarse en una experiencia de ecoturismo. Se trata de una pregunta de opción múltiple recogida en la Tabla 14, ya empleada por varios autores (Weaver y Lawton, 2002; Toselli, 2014; Ballesteros-Pelegrín, 2014; Marchena, 2015; Nel.lo y Llanes, 2016). Las tres actividades ecoturísticas (véase Tabla 14) de mayor interés, son: el senderismo, la observación de paisajes y la visita a parques naturales.

\section{Tabla Nº 14. Actividades ecoturísticas por país.}

\begin{tabular}{lc} 
Acampada en la naturaleza & 99 \\
Buceo marino, en ríos, lagos o ecobuceo & 41 \\
Ciclismo y mountain bike & 100 \\
Ecoarqueología & 21 \\
Escalada & 35 \\
Estudios de carácter científico o & 9 \\
expediciones científicas & \\
Exploración geológica y espeleológica & 31 \\
Montañismo & 191 \\
Observación de ballenas, tortugas & 32 \\
Observación de fauna & 173 \\
Observación de flora silvestre & 155 \\
Observación de paisajes naturales & 297 \\
Observación de aves & 109 \\
Paseos a caballo por espacios naturales & 41 \\
Pesca deportiva & 22 \\
Senderismo y excusiones por & 361 \\
sendas delimitadas & \\
Viajes o safaris fotográficos terrestres & 34 \\
y marítimos & \\
Visita a comunidades indígenas, & 30 \\
y conocimiento de culturales locales & \\
Visitas a parques naturales, nacionales & 314 \\
Visitas a bosques tropicales y & \\
arbustos indígenas & \\
Otros & 41 \\
\hline & 6
\end{tabular}

Con relación a la pertenencia a una asociación ecologista, animalista o protectora de animales, según se muestra en la Tabla 15, la mayoría de los encuestados, un $85,4 \%$, no pertenece a ninguna de estas entidades.
Tabla No 15. Pertenencia a una asociación ecologista o similar.

\begin{tabular}{lcc}
\hline & Frecuencia & Porcentaje \\
Sí & 118 & $14,6 \%$ \\
No & 691 & $85,4 \%$ \\
Total & 809 & $100 \%$ \\
\hline
\end{tabular}

Por país, con relación a los datos de pertenencia o no a una asociación ecologista o similar, encontramos que esta cifra es de $11,91 \%$.

Expectativas de los servicios que recibe del turismo ecológico

En relación las expectativas servicios del turismo ecológico, realizamos 5 cuestiones empleado una escala Likert de 5 puntos, la cual corresponde a una pregunta de opción múltiple, ya empleada por Lewis, Cheung y Jim (2014).

En el cuestionario se preguntó por cinco exceptivas de distintos servicios calidad del guía turístico, calidad de la información, importancia ecológica del destino, accesibilidad del destino e instalaciones del destino. Las puntuaciones medias de estas expectativas varían en función de las distintas afirmaciones. Por un lado, la importancia de la calidad del guía turístico es la menos valorada, posteriormente encontramos que el segundo elemento menos valorado son las instalaciones, el tercero la accesibilidad del servicio y en cuarto la calidad de la información. Por otro lado, el elemento más valorado, es la importancia ecológica del destino (véase tabla 16), igual que realizan otros estudios clásicos en países desarrollados, como el de Fennel y Eagles (1990) y Lewis et al., (2014). No obstante, difieren de éstos en los valores tan bajos que tiene la muestra española del papel de la importancia de la calidad de guía turístico, lo que lo aleja del planteamiento de Lewis et al., (2014). 
Tabla N 16. Expectativas sobre los servicios turísticos.

\begin{tabular}{lc}
\hline Iltem & Media \\
\hline $\begin{array}{l}\text { Lo que más me importa en mi viaje } \\
\text { ecológico es la calidad del guía turístico. }\end{array}$ & 3,34 \\
$\begin{array}{l}\text { Lo que más me importa en mi viaje } \\
\text { ecológico es la calidad de información }\end{array}$ & 3,85 \\
$\begin{array}{l}\text { que me proporciona el destino turístico. } \\
\text { Lo que más me importa en mi viaje }\end{array}$ & 4,04 \\
$\begin{array}{l}\text { ecológico es la importancia ecológica } \\
\text { del destino turístico. }\end{array}$ & \\
$\begin{array}{l}\text { Lo que más me importa en mi viaje } \\
\text { ecológico es la accesibilidad del } \\
\text { destino turístico. }\end{array}$ & 3,52 \\
$\begin{array}{l}\text { Lo que más me importa en mi viaje } \\
\text { ecológico son las instalaciones del } \\
\text { destino turístico. }\end{array}$ & 3,43 \\
\hline
\end{tabular}

\section{CONCLUSIONES}

En la actualidad la sociedad postmoderna anhela y estima acerca de la naturaleza en su tiempo de ocio, lo que está impulsando distintas formas de turismo alternativo, entre ellas el turismo ecológico o ecoturismo. Este incremento de número de visita supone una oportunidad para las empresas de turismo y ecoturismo de cara a sus actividades que conjugan crecimiento económico y protección de la naturaleza.

Conocer de mejor forma el perfil de las personas que hacen turismo ecológico o ecoturismo, y más concretamente de las expectativas en relación a determinados servicios, implica dotar de información a los operadores turísticos lo que facilita que estos puedan desarrollar planes, estrategias y campañas de difusión que mejoren entre otras cosas las experiencias turísticas de los ecoturistas antes, durante y después de su actividad turística, contribuyendo a la mejora de las experiencias de educación ambiental que esta tipología de ecoturismo lleva consigo.

La limitación cuantitativa de este estudio arroja la oportunidad de realizar nuevos estudios sobre las variables cualitativas de esta tipología de turismo y hacerlo a lo largo del tiempo más allá del carácter transeccional que posee un cuestionario. Por otro lado, es posible mejorar la calidad de la muestra empleada.
La investigación de los perfiles de ecoturismo de igual manera puede realizarse de forma global como en esta investigación y ser aplicada a territorios o ámbitos más concretos de investigación que pueden ser útiles para la industria turística.

\section{REFERENCIAS}

Beaumont, N. (2011). The third criterion of ecotourism: are ecotourists more concerned about sustainability than other tourists? Journal of Ecotourism, 10(2), 135-148.

Ceballos-Lascurain, H., (1987). Estudio de Perfectibilidad Socioeconómica del Turismo Ecológico y Anteproyecto arquitectónico y urbanístico del Centro de Turismo Ecológico de San Kalan, Quintana Roo. México: SEDUE.

Cobo Quesada, F. B., \& APARICIO SANCHEZ, M. D. S. (2014). Los parques nacionales españoles, catalizadores del turismo sostenible.

Das, M., \& Chatterjee, B. (2015). Ecotourism: A panacea or a predicament?. Tourism Management Perspectives, 14, 3-16.

Dias, R. (2007). Marketing ambiental. São Paulo: Editora Atlas.

Dolnicar, S. (2008). Market segmentation in tourism. Tourism management, analysis, behaviour and strategy, 129-150.

Dolnicar, S., \& Long, P. (2009). Beyond ecotourism: The environmentally responsible tourist in the general travel experience. Tourism Analysis, 14(4), 503513.

EUROPAR C (2016). EUROPARC-España. Anuario 2016 del estado de las áreas protegidas en España. Recuperado el 25 de julio de 2017 de : http://www. redeuroparc.org/system/files/shared/Publicaciones/Anuario_2016/anuario_2016_europarc-espana.pdf 
Fennell D, Eagles PF. 1990. Ecotourism in Costa Rica: a conceptual framework. J Parks Recreation Res. 8:23-34

Han, H., Hwang, J., y Lee, M. J. (2016). The value-beliefemotion-norm model: investigating customers eco-friendly behavior. Journal of Travel \& Tourism Marketing, 1-18.

INE (2016). Encuesta de movimientos turisticos de los Españoles Familitur (2016). Recuperado el 6 de septiembre de http://www.ine.es/dyngs/ INEbase/es/operacion.htm?c=Estadistica_C\&cid $=1254736176990 \&$ menu $=u$ uliDatos \&i $\mathrm{dp}=1254735576863$

Jiménez, B. L. H. (2009). Ecoturismo: oferta y desarrollo sistémico regional. Bogotá, CO: Ecoe Ediciones.

Juric, B., Cornwell, T. B., \& Mather, D. (2002). Exploring the usefulness of an Ecotourism Interest scale. Journal of Travel Research, 40(3), 259-269.

Kim, S. (2017). Study on possibilities of expansion of Japanese-Type ecotourism-focusing on nature-based daytrip tourists to ecotourism regions in Nabari City. European Journal of Tourism, Hospitality and Recreation, 8(1), 14-25.

Lewis T.O. Cheung \& C.Y. Jim (2014) Expectations and willingness-to-pay for ecotourism services in Hong Kong's conservation areas, International Journal of Sustainable Development \& World Ecology, 21:2, 149-159

Marchena Gómez, M. J. (2015). Turismo y desarrollo regional: el espacio del ecoturismo. Papers de Turisme, (11), 111-132.

Molina , A., Martin D.., Esteban Talaya, A. E., \& Díaz, E. (2007). Segmentación de la demanda turística: un análisis aplicado a un destino de turismo cultural. Revista de análisis turístico, (4).

Nel.Io M. y Llanes, C., (2016). Ecoturismo. Editorial UOC.
Neuts, B., Romão, J., Nijkamp, P., \& Shikida, A. (2016). Market segmentation and their potential economic impacts in an ecotourism destination: An applied modelling study on Hokkaido, Japan. Tourism Economics, 22(4), 793-808.

Pérez de las Heras, M. (1999). El ecoturismo como fórmula de desarrollo sostenible. en VIÑALS BLASCO, M. J. y BERNABÉ GARCÍA, A. Turismo en espacios naturales y rurales. Valencia, Universidad Politécnica de Valencia, Servicio de Publicaciones, pp. 103 129.

Pérez Hernández, I. Salinas Chávez, E, \& Perez Mijares, E. (2012). Consideraciones metodológicas para el desarrollo del ecoturismo a partir de las relaciones inter y transectoriales. Estudio de caso: Parque Nacional Viñales, Cuba. Gran Tour, (5), 8-26.

Puertas Cañaveral, I. (2006). Ecoturismo en las reservas de Biosfera: Análisis del ecoturista en Bañados del Este (Uruguay) y Cabo de Gata-Níjar (España). Estudios turísticos, (169-170), 183200

Puertas Cañaveral, I. (2006). Ecoturismo en las reservas de Biosfera: Análisis del ecoturista en Bañados del Este (Uruguay) y Cabo de Gata-Níjar (España). Estudios turísticos, (169-170), 183-200.

Puhakka, R., \& Siikamäki, P. (2012). Nature tourists' response to ecolabels in Oulanka PAN Park, Finland. Journal of Ecotourism, 11(1), 56-73.

Santesmases, M. (1999). Marketing: conceptos y estrategias. Ed. Piramide. Madrid.

Toselli, C. (2014). Turismo ecológico: aspectos generales sobre esta nueva tendencia. Signos Universitarios, 12(24).

Weaver, D. B., \& Lawton, L. J. (2007). Twenty years on: The state of contemporary ecotourism research. Tourism management, 28(5), 1168-1179. 